\section{OP55 EVALUATING COSTS OF ADVANCE CARE PLANNING: RESULTS FROM THE INTERNATIONAL ACTION STUDY}

${ }^{1}$ I Korfage, ${ }^{5} \mathrm{~S}$ Polinder, ${ }^{1} \mathrm{~A}$ van der Heide, ${ }^{2} \mathrm{~N}$ Preston, ${ }^{3} \mathrm{~J}$ van Delden, ${ }^{4} \mathrm{G}$ Miccinesi, ${ }^{5} \mathrm{U}$ Lunder, ${ }^{6} \mathrm{~K}$ Pollock, ${ }^{7} \mathrm{~L}$ Deliens, ${ }^{8} \mathrm{M}$ Groenvold, ${ }^{1} \mathrm{I}$ Rietjens. ${ }^{1}$ Department of Public Health, Erasmus MC, Rotterdam, The Netherlands; ${ }^{2}$ International Obsenvatory on End of Life Care, Division of Health Research, Lancaster University, Lancaster, UK; ${ }^{3}$ Julius Centre for Health Sciences and Primary Care, UMC Utrecht, Utrecht, The Netherlands; ${ }^{4}$ Clinical Epidemiology, Oncological network, prevention and research Institute (ISPRO), Florence, Italy; ${ }^{5}$ University Clinic of Respiratory and Allergic Diseases, Golnik, Slovenia; ${ }^{6}$ School of Health Sciences, University of Nottingham, Nottingham, UK; ${ }^{7}$ End-of-Life Care Research Group, Vrije Universiteit Brussel (VUB) \& Ghent University, Brussels, Belgium; ${ }^{8}$ Department of Public Health, University of Copenhagen, Copenhagen, Denmark

\subsection{6/spcare-2019-ACPICONGRESSABS.55}

Background Systematic evaluation of health care use and costs is important to identify the impact of advance care planning (ACP) programs. Such evaluations are currently scarce in Europe.

Methods Our study was performed in the context of the ACTION trial, a randomized controlled study to evaluate effects of the ACTION Respecting Choices (RC) ACP intervention in patients with advanced cancer in six European countries. We applied a healthcare perspective and identified hospital care use from hospital medical records for 1 year after study inclusion. Unit prices were calculated for all six countries separately. The unit price of the 'Respecting Choices' ACP intervention was determined with the micro-costing method, which is based on detailed assessments of all resources used.

Results Most intervention patients had one ACP conversation, one third had two. The average length was 90 minutes (standard deviation 45 minutes). Unit costs were comparable between countries for most interventions, with the exception of cancer-specific treatment. Most patients received chemotherapy, with a minority receiving surgery. Preliminary analyses showed similar patterns of health care use in both ACP and control groups: numbers of diagnostic procedures such as scans and biopsies were comparable; mean (range) length of hospital stay was 9 days $(0-63)$ and 8 days $(0-75)$ in intervention and control patients, respectively.

Conclusion Unit costs of health care interventions were remarkably comparable between countries. The ACTION RC ACP intervention, consisting of conversations by patiens, relatives, and facilitators, did not appear to affect hospital care use.

Funding EU FP7.

\section{OP56 THE COST-EFFECTIVENESS OF ADVANCE CARE PLANNING FOR OLDER ADULTS WITH END-STAGE KIDNEY DISEASE}

${ }^{1} \mathrm{M}$ Sellars*, ${ }^{2} \mathrm{~J}$ Clayton, ${ }^{1} \mathrm{~K}$ Detering, ${ }^{2} \mathrm{~A}$ Tong, ${ }^{1} \mathrm{D}$ Power, ${ }^{2} \mathrm{R}$ Morton. ${ }^{1}$ Austin Health, Melbourne, Australia; ${ }^{2}$ University of Sydney, Sydney, Australia

\subsection{6/spcare-2019-ACPICONGRESSABS.56}

Background We aimed to examine hospital costs and outcomes of a nurse-led ACP intervention compared with usual care in the last 12 months of life for older people with end-stage kidney disease (ESKD) managed with haemodialysis.

Methods A case-control study of ACP in adults with ESKD from a major tertiary hospital and a simulation of the natural history of decedents on dialysis, using hospital data, to model the effect of ACP on end-of-life care preferences. Outcomes were assessed in terms of patients' end-of-life treatment preferences being met or not, and costs included all hospital-based care. The cost-effectiveness of ACP was assessed by calculating an incremental cost-effectiveness ratio (ICER), expressed in dollars per additional case of end-of-life preferences being met. Robustness of model results was tested through sensitivity analyses.

Results The mean cost of ACP was AUD\$519 per patient. The mean hospital costs of care in last 12 months of life were $\$ 100,579$ for those who received ACP versus $\$ 87,282$ for those who did not. The proportion of patients in the model who received end-of-life care according to their preferences was higher in the ACP group compared with usual care $(68 \%$ vs. $24 \%)$. The incremental cost per additional case of end-of-life preferences being met was $\$ 28,421$. The greatest influence on the cost-effectiveness of ACP was the probability of dying in hospital following dialysis withdrawal, and costs of acute care.

Conclusions Our model suggests nurse-led ACP leads to receipt of patient preferences for end-of-life care, and may represent good value for money.

\section{OP57 ADVANCE CARE PLANNING FOR FRAIL OLDER ADULTS: FINDINGS ON COSTS IN A CLUSTER RANDOMISED CONTROLLED TRIAL}

${ }^{1} \mathrm{~A}$ Overbeek*, ${ }^{1} \mathrm{~S}$ Polinder, ${ }^{1} \mathrm{~J}$ Haagsma, ${ }^{2} \mathrm{P}$ Billekens, ${ }^{1} \mathrm{~K}$ De Nooijer, ${ }^{3} \mathrm{~B}$ Hammes, ${ }^{1} \mathrm{D}$ Muliaditan, ${ }^{1} \mathrm{~A}$ van der Heide, 'J Rietjens, 'I Korfage. 'Erasmus MC, Rotterdam, Netherlands; ' Laurens, the Netherlands; ${ }^{3}$ Respecting Choices, C-TAC Innovations, USA

\subsection{6/spcare-2019-ACPICONGRESSABS.57}

Background Advance Care Planning aims at improving alignment of care with patients' preferences. This may affect costs of medical care.

Aim To determine the costs of an Advance Care Planning programme and its effects on the costs of medical care and on concordance of care with patients' preferences.

Design/settings/participants In a cluster randomised trial, 16 residential care homes were randomly allocated to the intervention group, where frail, older participants were offered facilitated Advance Care Planning conversations or to the control group. We calculated variable costs of Advance Care Planning per participant including personnel and travel costs of facilitators. Furthermore, we assessed participants' healthcare use during 12 months applying a broad perspective (including medical care, inpatient days in residential care homes, home care) and calculated costs of care per participant. Finally, we investigated whether treatment goals were in accordance with preferences. Analyses were conducted for 97 participants per group. Trial registration number: NTR4454.

Results Average variable Advance Care Planning costs were $€ 76$ per participant. The average costs of medical care were not significantly different between the intervention and control group $(€ 2360$ vs $€ 2235$, respectively, $p=0.36$ ). Costs of inpatient days in residential care homes $(€ 41,551$ vs $€ 46,533)$ and of home care $(€ 14,091$ vs $€ 17,361)$ were not significantly different either. Concordance of care with preferences could not be assessed since treatment goals were often not recorded. 